\title{
Antibiotic resistance pattern of uropathogenic Escherichia coli isolated from children with symptomatic urinary tract infection in Moscow, Russia
}

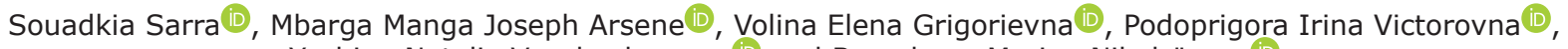 \\ Yashina Natalia Vyacheslavovna(i) and Borechova Marina Nikolaïevna (iD) \\ Department of Microbiology and Virology, Institute of Medicine, RUDN University, Moscow, Russia. \\ Corresponding author: Mbarga Manga Joseph Arsene, e-mail: josepharsenembarga@yahoo.fr \\ Co-authors: SS: sarah1sdk@gmail.com, VEG: volina-eg@rudn.ru, PIV: podoprigora-iv@rudn.ru; \\ YNV: yashina-nv@rudn.ru; BMN: borekhova-mn@rudn.ru \\ Received: 30-06-2021, Accepted: 13-09-2021, Published online: 23-11-2021
}

doi: www.doi.org/10.14202/IJOH.2021.212-219 How to cite this article: Sarra S, Arsene MMJ, Grigorievna VE, Victorovna PI, Vyacheslavovna YN, Nikolaïevna BM (2021) Antibiotic resistance pattern of uropathogenic Escherichia coli isolated from children with symptomatic urinary tract infection in Moscow, Russia, Int. J. One Health, 7(2):212-219.

\begin{abstract}
Background and Aim: Uropathogenic Escherichia coli (UPEC) is commonly involved in urinary tract infections (UTIs), which are generally treated with antibiotics. However, the emergence of multidrug-resistant (MDR) strains of UPEC has made the treatment difficult. There is thus a need to continuously assess their sensitivity to antibiotics. This study aimed to determine the antibiotic resistance patterns and MDR phenotypes of UPEC strains isolated from children diagnosed with UTIs at the Russian Children's Clinical Hospital in Moscow, Russia.
\end{abstract}

Materials and Methods: Kirby-Bauer's disc diffusion method was used to study the sensitivity to antibiotics of 106 UPEC isolates from urine specimens from children (aged from 9 months to 18 years old) diagnosed with UTIs. The results were interpreted in accordance with theClinical and Laboratory Standards Institute guidelines and the correlations of variables with the degree to which each antibiotic inhibited the UPEC strains in terms of diameter on the disc were determined using Spearman's rank correlation test. A t-test and principal component analysis were performed to visualize the correlations of the susceptibility of UPEC to antibiotics with the age and sex of the patients. Statistical significance was set at $\mathrm{p} \leq 0.05$.

Results: Among the 106 UPEC strains tested, none (0\%) showed resistance to fosfomycin (FO), while 84 (79.2\%) were resistant $(\mathrm{R})$ to at least one antibiotic. The highest rates of resistance were observed to amoxicillin (69.8\%), ampicillin $(62.3 \%)$, cefazolin $(39.6 \%)$, trimethoprim (TR) $(37.7 \%)$, ceftriaxone $(34.9 \%)$, and tetracycline (33.0\%). Interestingly, $22(20.8 \%)$ strains were R to imipenem. UPEC isolates from males aged 1-6 years were more R to antibiotics than those from the other groups, with the exception of TR, to which UPEC isolates from females aged 13-18 years old were less sensitive (S). The multidrug-resistance (MDR) index ranged between 0.00 and 0.75 and we found that more than a quarter of $\operatorname{UPEC}(31 / 106)$ had an MDR index $\geq 0.5$ and only $22(20.7 \%)$ strains were $\mathrm{S}$ to all antibiotics tested (MDR index $=0)$. Finally, Spearman's rank correlation test showed that, with the exception of FO, there were correlations between the inhibition diameters of all other antibiotics.

Conclusion: FO is the only antibiotic to which all UPECs were S and may be suggested as the first line of treatment for UPEC. Further research is needed to continue monitoring antibiotic resistance and to investigate the genetic features associated with such resistance observed in this study.

Keywords: antibiotics, multidrug resistance, urinary tract infections, uropathogenic Escherichia coli.

\section{Introduction}

Urinary tract infections (UTIs) are among the most common bacterial infections, affecting around 150-250 million people each year worldwide [1]. These infections account for $75 \%$ of infections in community settings and 50-65\% of those in health-care settings [2,3]. In uncomplicated UTIs (50-90\%), strains of uropathogenic Escherichia coli (UPEC) are the most common

Copyright: Sarra, et al. This article is an open access article distributed under the terms of the Creative Commons Attribution 4.0 International License (http://creativecommons.org/licenses/ by/4.0/), which permits unrestricted use, distribution, and reproduction in any medium, provided you give appropriate credit to the original author(s) and the source, provide a link to the Creative Commons license, and indicate if changes were made. The Creative Commons Public Domain Dedication waiver (http:// creativecommons.org/ publicdomain/zero/1.0/) applies to the data made available in this article, unless otherwise stated. organisms seen $[4,5]$. In such cases, these strains diverge from their status as part of the commensal intestinal flora and colonize the urinary tract, exhibiting various virulence factors, which allow them to infect that tract [6].

It is estimated that $40 \%$ of women and $12 \%$ of men experience a minimum of one symptomatic UTI episode during their lifetime, and $27-48 \%$ of affected women suffer from recurrent UTIs [7,8]. UPEC is the most common group of bacterial pathogens causing UTIs in children [9] and leads to the recurrence of infection in 10-30\% of cases [10]. Infections caused by UPECs are generally treated with antibiotics. However, in recent years, several complications have been observed in the treatment and management of patients with this pathology due to the emergence and spread of antibiotic resistance $[11,12]$. 
Furthermore, multidrug-resistant $(\mathrm{R})$ organisms can cause an increase in UTIs in children that are difficult to treat [13]. The increasing prevalence of $R$ Enterobacteriaceae causing UTIs in children has been widely recorded [14].

Consequently, it has become necessary to regularly assess the antibiotic sensitivity of UPEC strains to establish their resistance profile and to monitor the evolution of antibiotic resistance in specific geographical areas. Thus, this study was conducted to evaluate the antibiotic sensitivities of UPEC strains isolated from children diagnosed with UTIs at the Russian Children's Clinical Hospital in Moscow.

\section{Materials and Methods}

\section{Ethical approval and informed consent}

After retrieval of relevant information from the referral, all isolates were anonymized, with only the age and sex being retained. Therefore, no ethical approval was necessary.

\section{Study period and location}

This study was performed from November 2017 to June 2018 at the Russian children's clinical hospital, Moscow, Russia, and in the Department of Microbiology and Virology of the People's Friendship University of Russia.

\section{Collection of isolates}

The 106 strains of E. coli used in the present study were isolated from urine specimens taken from patients (children aged 9 months to 18 years old) with symptomatic and laboratory-confirmed UTIs. The bacteria were not repetitive since only one bacterium was considered per patient. These bacteria were isolated and identified in the laboratory of the Russian Children's Clinical Hospital and transferred to the research laboratory of the Department of Microbiology and Virology of People's Friendship University of Russia, where they were kept frozen at $-80^{\circ} \mathrm{C}$.

\section{Culture conditions}

All of the cultures were performed on Brain Heart Infusion Broth (BHIB) (HiMedia ${ }^{\mathrm{TM}}$ Laboratories Pvt. Ltd., India) and Muller Hinton Agar (MHA HiMedia ${ }^{\mathrm{TM}}$ ) and incubated aerobically at $37^{\circ} \mathrm{C}$ for 18-24 h.

\section{Antimicrobial susceptibility testing}

The antimicrobial resistance pattern was determined bythe modified Kirby-Bauer's disc diffusion method, as described by Manga et al. [15]. Briefly, after bringing the bacteria at room temperature, they were cultured at $37^{\circ} \mathrm{C}$ for $24 \mathrm{~h}$ in sterile BHIB. A total of $1.5 \mathrm{~mL}$ of each overnight culture was centrifuged (Eppendorf Centrifuge $5415 \mathrm{R}$ ) for $10 \mathrm{~min}$ at 3000 RCF and the pellet was collected, washed 3 times with phosphate-buffered saline, and resuspended in $5 \mathrm{~mL}$ of physiological water to obtain a concentration equivalent to $0.5 \mathrm{McF}$ arland. Then, $100 \mu \mathrm{L}$ of the culture was plated on Muller Hinton Agar and the antibiotic discs were placed aseptically using a dispenser. The following 12 antibiotics were used: Amoxicillin (AMC), $30 \mu \mathrm{g} / \mathrm{disc}$; ampicillin (AMP), $25 \mu \mathrm{g} / \mathrm{disc}$; cefazolin (CZ), $30 \mu \mathrm{g} / \mathrm{disc}$; CZ/clavulanic acid (CAC), 30/10 per disc; ceftazidime (CAZ), $30 \mu \mathrm{g} / \mathrm{disc}$; ceftriaxone (CTR), $30 \mu \mathrm{g} / \mathrm{disc}$; ciprofloxacin (CIP), $30 \mu \mathrm{g} /$ disc; fosfomycin (FO), $200 \mu \mathrm{g} / \mathrm{disc}$; imipenem (IMP), $10 \mu \mathrm{g} /$ disc; nitrofurantoin (NIT), $200 \mu \mathrm{g} / \mathrm{disc}$; tetracycline (TE), $30 \mu \mathrm{g} / \mathrm{disc}$; and trimethoprim (TR), $30 \mu \mathrm{g} /$ disc.

\section{Interpretation of results and data analysis}

After $18-24 \mathrm{~h}$ of incubation at $37^{\circ} \mathrm{C}$, the inhibition diameters were measured and interpreted in accordance with the Clinical and Laboratory Standards Institute [16]. R, intermediate (I), and sensitive (S) interpretations were obtained automatically using algorithms written in Excel software (Microsoft Office 2016 MSO version 16.0.13628.20128[32 bits], USA) with the parameters described in Table-1 $[15,16]$. The software Minitab 18 was used to perform Spearman's rank correlation test between inhibition diameters and the statistical significance was set at $\mathrm{p} \leq 0.05$. A t-test and principal component analysis (PCA) were carried out using XLSTAT 2020 statistical software (Addinsof Inc., New York, USA). PCA was used to visualize the correlations of the susceptibility of UPEC to antibiotics with the age and sex of the patients from whom the bacteria were isolated.

\section{Results}

In this study, the 106 UPEC tested were previously isolated from the urine of children $(n=106)$ aged 9 months to 18 years old with laboratory-confirmed UTIs. The median age was 5.5 years and the maleto-female ratio was $0.68: 1$. To facilitate data analysis and interpretation of the results, the subjects were divided into six groups based on sex and age ranges of 1-6 years, 7-12 years, and 12-18 years. All results involving sex and age were expressed as intragroup prevalence, that is, as a percentage relative to the group considered.

Figure-1 shows the sensitivity of the isolated UPEC to antibiotics. Out of the 1272 antibiograms performed, we observed $77(29.6 \%)$ R cases, $109(8.6 \%)$ I cases, and $786(61.8 \%) \mathrm{S}$ cases. Isolated UPECs exhibited the highest resistance to AMC (69.8\%), AMP (62.3\%), CZ (39.6\%), TR (37.7\%), CTR (34.9\%), and TE (33.0\%). Interestingly, no UPEC was R to FO and, unlike cefazoline, CZ/CAC was highly active in $90.6 \%$ of UPEC. In addition, antibiotics such as NIT, CIP, and IMP were also very active, with susceptibility rates of $80.2 \%, 73.6 \%$, and $70.8 \%$, respectively. Furthermore, Figures- 2 and 3 present the overall prevalence of the susceptibility of UPEC to antibiotics depending on the age and sex of the children from whom the bacteria were isolated.

As presented in Figure-2, UPEC isolated from males aged 1-6 years were more R to AMP, amoxicillin, CAZ, and cefazoline. Those isolated from males aged 13-18 years were also R to AMP and amoxicillin, 
Table 1: Interpretation criteria for antibiotic sensitivity of enterobacteria $[15,16]$.

\begin{tabular}{lcccccccccccc}
\hline Interpretation & \multicolumn{10}{c}{ Antibiotics/limits of inhibition diameters (mm) } \\
\cline { 2 - 14 } & CIP & CZ & CAZ & AMC & CTR & TR & TE & NIT & AMP & IMP & CAC & FO \\
\hline $\mathrm{R}$ & $\mathrm{d} \leq 15$ & $\mathrm{~d} \leq 14$ & $\mathrm{~d} \leq 14$ & $\mathrm{~d} \leq 13$ & $\mathrm{~d} \leq 13$ & $\mathrm{~d} \leq 13$ & $\mathrm{~d} \leq 14$ & $\mathrm{~d} \leq 13$ & $\mathrm{~d} \leq 13$ & $\mathrm{~d} \leq 13$ & $\mathrm{~d} \leq 14$ & $\mathrm{~d} \leq 12$ \\
$\mathrm{I}$ & $16-20$ & $15-17$ & $15-17$ & $14-17$ & $14-20$ & $14-15$ & $15-18$ & $14-17$ & $14-16$ & $14-15$ & $15-17$ & $13-15$ \\
$\mathrm{~S}$ & $\mathrm{~d} \geq 21$ & $\mathrm{~d} \geq 18$ & $\mathrm{~d} \geq 18$ & $\mathrm{~d} \geq 18$ & $\mathrm{~d} \geq 21$ & $\mathrm{~d} \geq 16$ & $\mathrm{~d} \geq 19$ & $\mathrm{~d} \geq 18$ & $\mathrm{~d} \geq 17$ & $\mathrm{~d} \geq 16$ & $\mathrm{~d} \geq 18$ & $\mathrm{~d} \geq 17$ \\
\hline
\end{tabular}

$\mathrm{AMC}=$ Amoxycillin, $\mathrm{AM}=$ Ampicillin, $\mathrm{CZ}=$ Cefazolin, $\mathrm{CAC}=$ Cefazolin/clavulanic acid, CAZ=Ceftazidime, CTR=Ceftriaxone, $\mathrm{CIP}=$ Ciprofloxacin, $\mathrm{FO}=$ Fosfomycin, IMP=Imipenem, NIT=Nitrofurantoin, $T E=$ Tetracyclin, $\mathrm{TR}=$ Trimethoprim

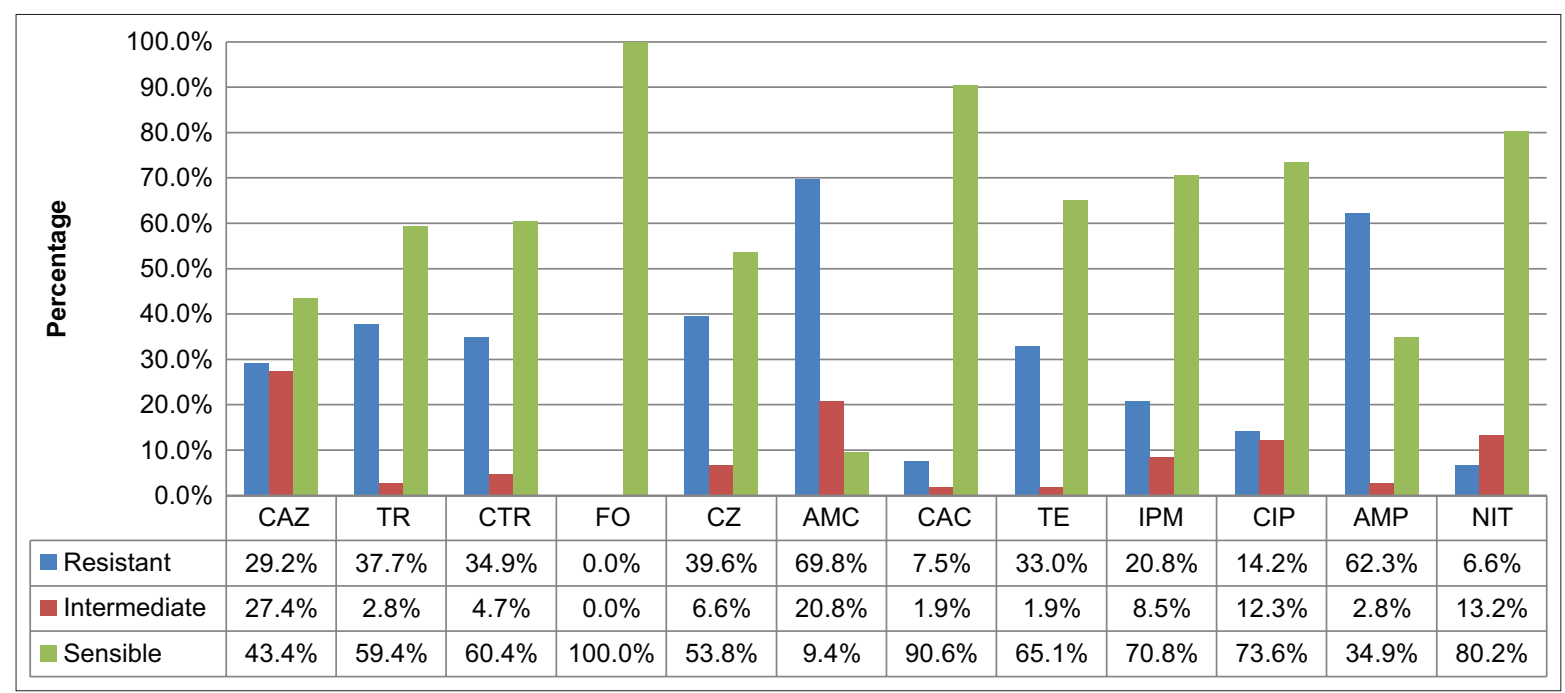

Figure-1: Sensitivity to antibiotics of the 106 uropathogenic Escherichia coli isolated. R=Resistant, I=Intermediate, $\mathrm{S}=$ Sensitive, $\mathrm{AMC}=$ Amoxycillin, $\mathrm{AMP}=$ Ampicillin, $\mathrm{CZ}=$ Cefazolin, $\mathrm{CAC}=$ Cefazolin/clavulanic acid, $C A Z=C e f t a z i d i m e$, $\mathrm{CTR}=$ Ceftriaxone, $\quad \mathrm{CIP}=$ Ciprofloxacin, $\quad \mathrm{FO}=$ Fosfomycin, IMP=Imipenem, NIT=Nitrofurantoin, TE=Tetracyclin, $\mathrm{TR}=$ Trimethoprim

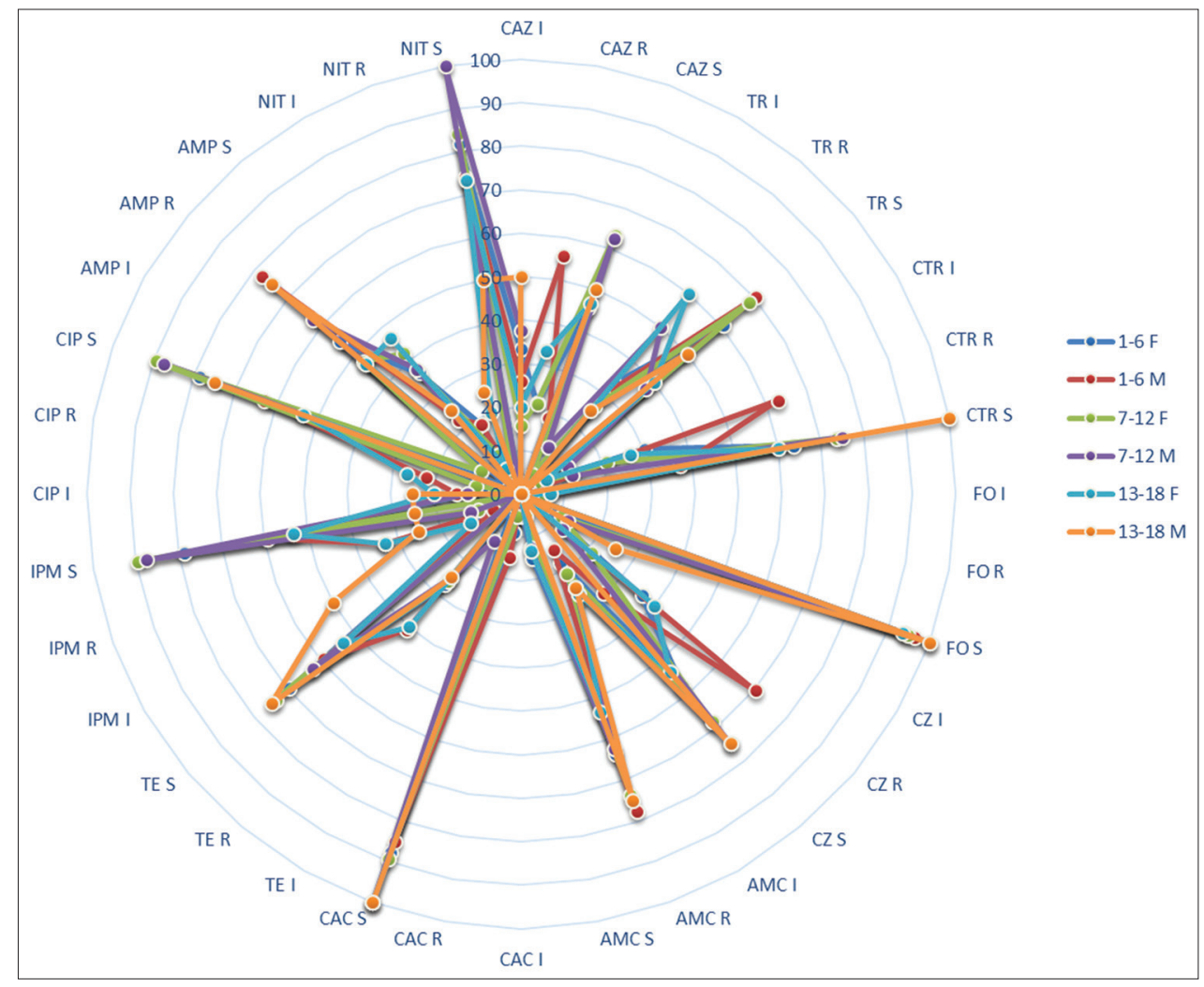

Figure-2: Overall prevalence of the susceptibility of uropathogenic Escherichia coli to antibiotics depending on the age and sex of the children from whom the bacteria were isolated. $\mathrm{R}=$ Resistant, $\mathrm{I}=$ Intermediate, $\mathrm{S}=$ Sensitive, $\mathrm{AMC}=\mathrm{Amoxycillin}$, $\mathrm{AMP}=$ Ampicillin, $\mathrm{CZ}=$ Cefazolin, $\mathrm{CAC}=$ Cefazolin/clavulanic acid, CAZ=Ceftazidime, CTR=Ceftriaxone, CIP=Ciprofloxacin, $\mathrm{FO}=$ Fosfomycin, IMP=Imipenem, NIT=Nitrofurantoin, TE=Tetracycline, TR=Trimethoprim. 
but very $\mathrm{S}$ to $\mathrm{CTR}$ and $\mathrm{CZ} / \mathrm{CAC}$. Moreover, UPEC isolated from children aged 7-12 years were all $\mathrm{S}$ to IMP, CIP, NIT, and FO. As shown in Figure-3, UPEC strains isolated from females aged 13-18 years old were the most $\mathrm{R}$ to TR.

In addition, as observed in Table-2, Spearman's rank correlation test showed that, with the exception of FO, there were strong correlations among the sensitivities of UPEC to 11 other antibiotics, with probabilities ranging from 0.000 to $0.035(\mathrm{p} \leq 0.05)$ and Spearman's coefficients ranging from 0.241 to 0.917 . The strongest correlation was observed between susceptibility to IMP and susceptibility to CIP ( $p<0.000$ and Spearman's coefficient $=0.917$ ).

To visualize the associations among age, sex, and susceptibility of UPEC to antibiotics, PCA was performed. Figure- 4 shows the distribution of the susceptibility to antibiotics of the tested UPEC, age, and sex in an F1 $\times F 2$ system. UPEC isolated from 13 to 18-year-old females and 1-6-year-old children were globally strongly correlated with resistance to TE, CAZ, amoxicillin and to IMP, TR, cefazoline, while those from 7 to 12 -year-old children were more correlated with sensitivity to most antibiotics used. Finally, as shown in Table-3, the multidrug resistance (MDR) index was obtained for each strain. We found that more than a quarter of UPEC $(31 / 106)$ had an MDR index $\geq 0.5$ and only $22(20.7 \%)$ strains were $S$ to all of the antibiotics tested (MDR index=0).

\section{Discussion}

Due to limited resources, this study focused only on analyzing the phenotype of antibiotic sensitivity of UPEC isolated from children diagnosed with UTIs. The selected antibiotics were those commonly used for treating UTIs in Russia.

In this study, the high resistance observed against AMP and amoxicillin was in accordance with previously reported results [17-19]. This high rate can be explained by the fact that these antibiotics are regularly used as first-line treatments as well as self-medication in the treatment of common bacterial infections $[20,21]$. These practices lead to the recurrent exposure of normal microbiota (including E. coli) to these antibiotics, which may prompt adaptation of the strains and thus antibiotic resistance [20,22-24]. Paschke et al. [22] reported that recent antimicrobial exposure is associated with antimicrobial-R UTIs among pediatric outpatients and that the magnitude of this association decreases with time since exposure. In their study, out of 533 children who had a first UTI, those exposed to amoxicillin within 30 days and 31-60 days before the UTI were associated with both AMP and amoxicillin-clavulanate resistance [22]. They also reported that there was no association between exposure to other antimicrobial agents and resistance to any of the antimicrobial agents [22]. Therefore, the high resistance to AMP and amoxicillin in UPEC isolated from children aged 1-6 years compared with the levels of other groups, which seemed unusual, could be explained by the previous study [22]. Unfortunately, in our study, information on the drugs taken by these children before their infection was not available, limiting our ability to perform comparisons with the data reported in the literature.

Otherwise, contrary to many studies $[12,25,26]$, $20.8 \%$ of our isolates were R to IMP. Shirani et al. [17] reported that certain UPEC acquire genes encoding extended-spectrum beta-lactamases. These enzymes destroy the $\beta$-lactam ring in the antibiotic structure of many antibiotics including carbapenems [17]. The emergence of carbapenem-R UPEC makes treatment of these infections increasingly challenging $[26,27]$.

Moreover, we found that UPEC strains isolated from females aged 13-18 years old were the most

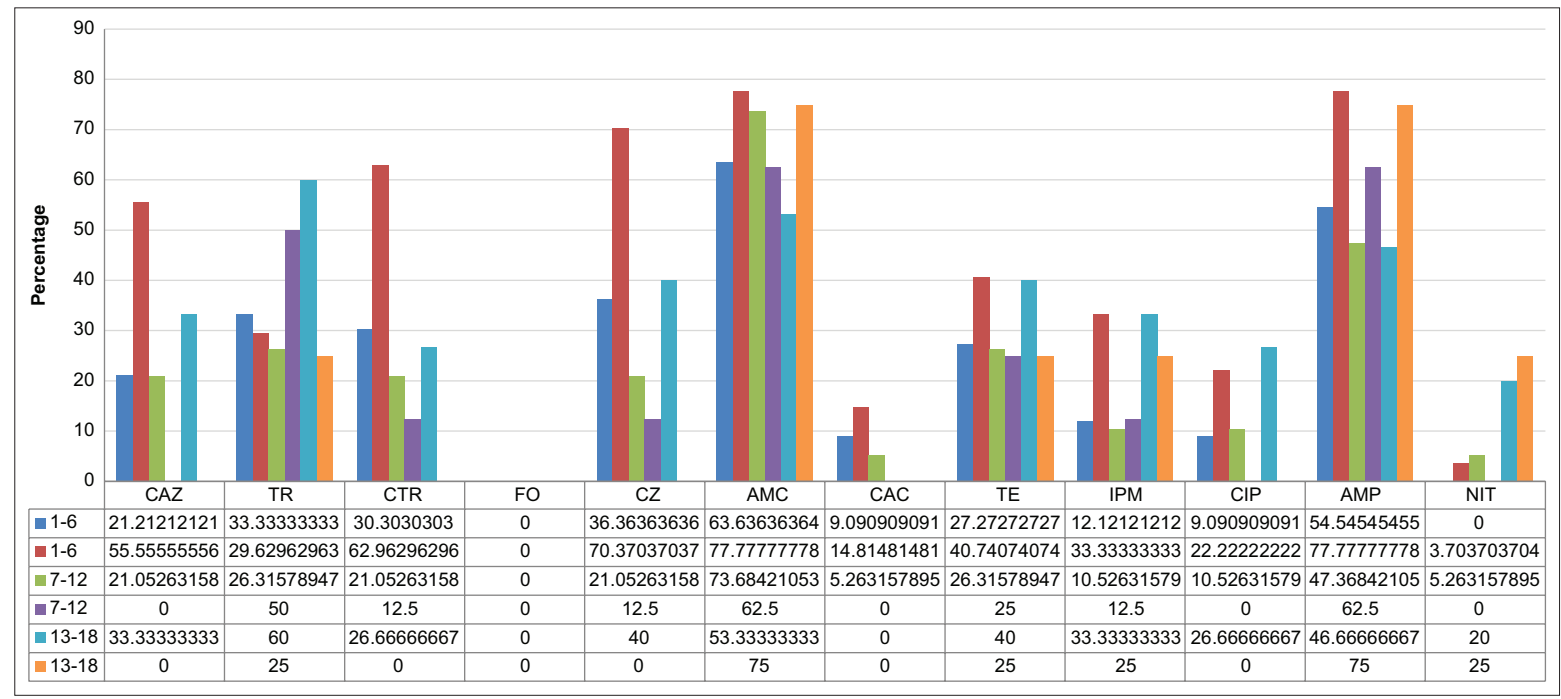

Figure-3: Prevalence of the resistance of uropathogenic Escherichia coli to antibiotics depending on the age and sex of the children from whom the bacteria were isolated. $\mathrm{R}=$ Resistant, $\mathrm{I}=$ Intermediate, $\mathrm{S}=$ Sensitive, $\mathrm{AMC}=\mathrm{Amoxycillin}$, $\mathrm{AMP}=$ Ampicillin, $\mathrm{CZ}=$ Cefazolin, $\mathrm{CAC}=$ Cefazolin/clavulanic acid, CAZ=Ceftazidime, CTR=Ceftriaxone, CIP=Ciprofloxacin, $\mathrm{FO}=$ Fosfomycin, IMP=Imipenem, NIT=Nitrofurantoin, TE=Tetracycline, TR=Trimethoprim. 
Table 2: Spearman rank correlation test between the susceptibility to antibiotics of the uropathogenic Escherichia coli isolated. Statistical significance was considered at $P<0.05$.

\begin{tabular}{|c|c|c|c|c|c|c|c|c|c|c|c|}
\hline & CAZ & TR & CTR & FO & $\mathbf{C Z}$ & AMC & CAC & TE & IPM & CIP & AMP \\
\hline \multicolumn{12}{|l|}{ TR } \\
\hline Spearman coef & 0.106 & & & & & & & & & & \\
\hline p-value & 0.024 & & & & & & & & & & \\
\hline \multicolumn{12}{|l|}{ CTR } \\
\hline Spearman coef & 0.852 & 0.180 & & & & & & & & & \\
\hline p-value & 0.000 & 0.065 & & & & & & & & & \\
\hline \multicolumn{12}{|l|}{ FO } \\
\hline Spearman coef & 0.015 & 0.156 & 0.013 & & & & & & & & \\
\hline p-value & 0.881 & 0.110 & 0.897 & & & & & & & & \\
\hline \multicolumn{12}{|l|}{$C Z$} \\
\hline Spearman coef & 0.772 & 0.205 & 0.794 & 0.075 & & & & & & & \\
\hline p-value & 0.000 & 0.035 & 0.000 & 0.442 & & & & & & & \\
\hline \multicolumn{12}{|l|}{ AMC } \\
\hline Spearman coef & 0.399 & 0.341 & 0.436 & 0.029 & 0.674 & & & & & & \\
\hline p-value & 0.000 & 0.000 & 0.000 & 0.765 & 0.000 & & & & & & \\
\hline \multicolumn{12}{|l|}{ CAC } \\
\hline Spearman coef & 0.822 & 0.137 & 0.764 & -0.021 & 0.730 & 0.393 & & & & & \\
\hline p-value & 0.000 & 0.162 & 0.000 & 0.831 & 0.000 & 0.000 & & & & & \\
\hline \multicolumn{12}{|l|}{ TE } \\
\hline Spearman coef & 0.265 & 0.456 & 0.267 & 0.101 & 0.382 & 0.403 & 0.232 & & & & \\
\hline p-value & 0.006 & 0.000 & 0.006 & 0.304 & 0.000 & 0.000 & 0.017 & & & & \\
\hline \multicolumn{12}{|l|}{ IPM } \\
\hline Spearman coef & 0.435 & 0.419 & 0.463 & 0.008 & 0.524 & 0.444 & 0.493 & 0.297 & & & \\
\hline p-value & 0.000 & 0.000 & 0.000 & 0.939 & 0.000 & 0.000 & 0.000 & 0.002 & & & \\
\hline \multicolumn{12}{|l|}{ CIP } \\
\hline Spearman coef & 0.393 & 0.453 & 0.414 & -0.001 & 0.450 & 0.337 & 0.424 & 0.307 & 0.917 & & \\
\hline p-value & 0.000 & 0.000 & 0.000 & 0.991 & 0.000 & 0.000 & 0.000 & 0.001 & 0.000 & & \\
\hline \multicolumn{12}{|l|}{ AMP } \\
\hline Spearman coef & 0.492 & 0.345 & 0.507 & 0.045 & 0.733 & 0.808 & 0.458 & 0.373 & 0.469 & 0.414 & \\
\hline p-value & 0.000 & 0.000 & 0.000 & 0.649 & 0.000 & 0.000 & 0.000 & 0.000 & 0.000 & 0.000 & \\
\hline \multicolumn{12}{|l|}{ NIT } \\
\hline Spearman coef & 0.190 & 0.299 & 0.241 & 0.005 & 0.178 & 0.096 & 0.268 & 0.251 & 0.399 & 0.369 & 0.067 \\
\hline p-value & 0.051 & 0.002 & 0.013 & 0.956 & 0.068 & 0.326 & 0.006 & 0.009 & 0.000 & 0.000 & 0.494 \\
\hline
\end{tabular}

$\mathrm{AMC}=$ Amoxycillin, $\mathrm{AM}=$ Ampicillin, $\mathrm{CZ}=$ Cefazolin, $\mathrm{CAC}=$ Cefazolin/clavulanic acid, CAZ=Ceftazidime, CTR=Ceftriaxone, $\mathrm{CIP}=$ Ciprofloxacin, $\mathrm{FO}=$ Fosfomycin, IMP=Imipenem, NIT=Nitrofurantoin, $\mathrm{TE}=$ Tetracyclin, $\mathrm{TR}=$ Trimethoprim

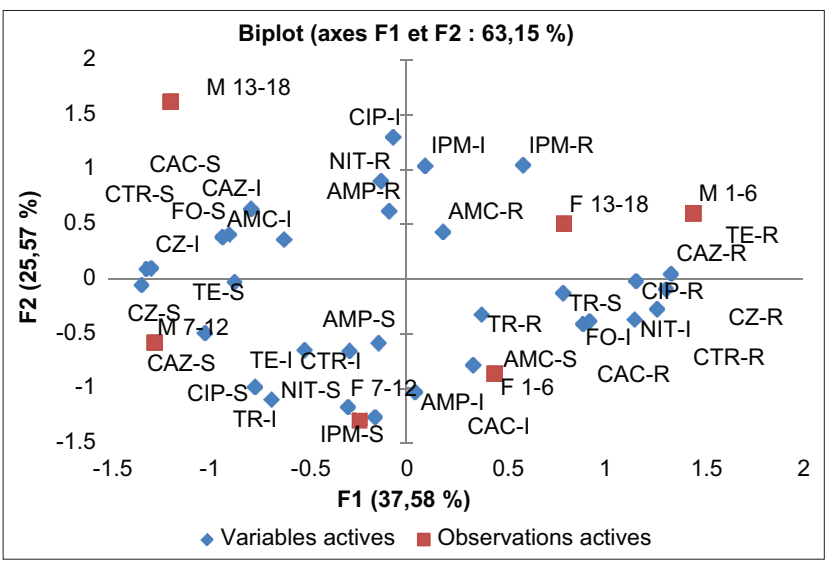

Figure-4: Principal component analysis of age, sex ( $F=$ female; $M=$ male) and susceptibility ( $R=$ Resistant; I=Intermediate; $\mathrm{S}=$ Sensitive) to $\mathrm{AMC}=$ Amoxycillin, AMP=Ampicillin, $\quad \mathrm{CZ}=$ Cefazolin, $\quad \mathrm{CAC}=$ Cefazolin $/$ clavulanic acid, CAZ=Ceftazidime, CTR=Ceftriaxone, $\mathrm{CIP}=$ Ciprofloxacin, $\mathrm{FO}=$ Fosfomycin, $\mathrm{IMP}=$ Imipenem, NIT=Nitrofurantoin, $T E=$ Tetracycline, $T R=$ Trimethoprim.

$\mathrm{R}$ to TR. Trimethoprim-sulfamethoxazole is one of the most recommended antibiotics for treating acute uncomplicated UTIs $[28,29]$, but resistance to this antibiotic is increasing in the management of UTIs and was also reported elsewhere [30]. Eliopoulos and
Huovinen [31] reported that bacterial resistance to TMP is mediated by the following five main mechanisms: (1) The permeability barrier and/or efflux pumps, (2) naturally insensitive target enzymes, (3) regulatory changes in the target enzymes, (4) mutational or recombinational changes in the target enzymes, and (5) the acquisition of resistance by drug- $\mathrm{R}$ target enzymes. In UPEC, the adaptation resulting from recurrent exposure to the antimicrobial is usually one of the most evoked ways of explaining this growth in resistance. Nevertheless, studies have reported that resistance alleles such as sull, sul2, $s u l 3$, and $d f r$ along with Type 1 and Type 2 integrons were actively involved in the horizontal transmission of TR resistance [32]. In a study conducted by Blahna et al. [32] to describe the distribution of TR-S resistance genes and the role of horizontal gene transfer and clonal expansion in recent increases of antibiotic resistance rates among UPEC in Europe and Canada, the authors found that $d f r A l$ was the most common in Europe. However, unfortunately in our study, no analysis was performed on the genetic features associated with resistance.

Interestingly, all of our isolates were susceptible to FO, another antibiotic frequently recommended for 
Table 3: MDR index and resistance pattern of the isolated UPEC.

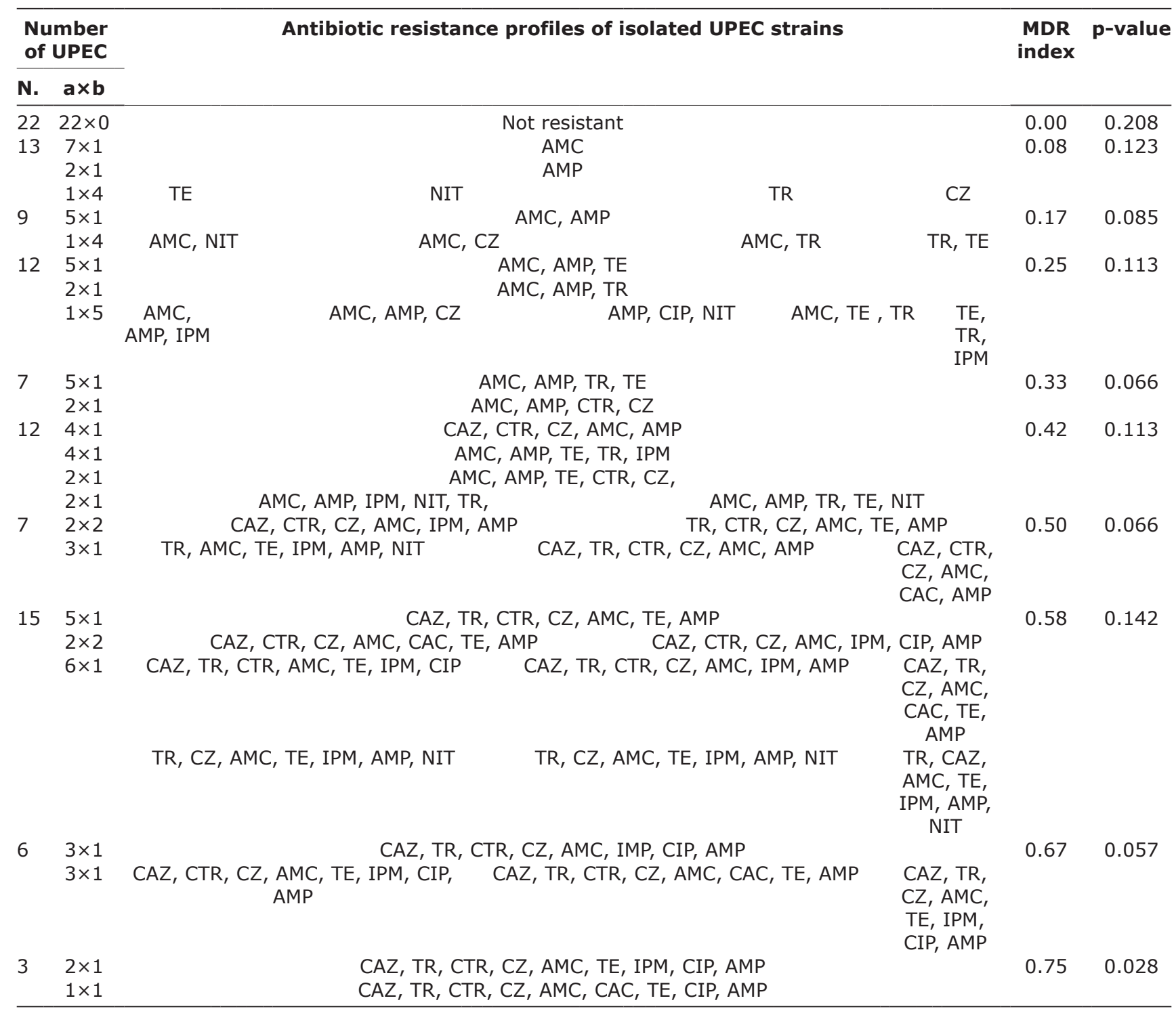

$a=$ Number of UPEC strains. $b=$ Number of resistance phenotypes in each UPEC strain. $N=$ Total number of resistance phenotypes in each group in the studied UPEC strains. $P=$ Probability coefficient. $\mathrm{R}=$ Resistant; $\mathrm{I}=$ Intermediate; $\mathrm{S}=$ Sensitive, $\mathrm{AMC}=$ Amoxycillin, $\mathrm{AMP}=$ Ampicillin, $\mathrm{CZ}=$ Cefazolin, $C A C=$ Cefazolin/clavulanic acid, CAZ=Ceftazidime, $\mathrm{CTR}=$ Ceftriaxone, $\mathrm{CIP}=$ Ciprofloxacin, $\mathrm{FO}=$ Fosfomycin, IMP=Imipenem, NIT=Nitrofurantoin, TE=Tetracyclin, $\mathrm{TR}=$ Trimethoprim, UPEC=Uropathogenic Escherichia coli

the management of UTIs. This finding is in accordance with the study of Hirsch et al. [33], in which all UPEC strains investigated were susceptible to FO [33]. Likewise, Kresken et al. [34] reported that less than $1.5 \%$ of UPEC were $\mathrm{R}$ to this antibiotic [28]. This suggests that FO could be considered as a firstline treatment for UTIs; indeed, this antibiotic is currently approved for use in some European countries as a treatment for uncomplicated UTIs caused by E. coli [35]. Although FO remains the preferred agent for treating uncomplicated cystitis, its use may be limited in many patients due to low creatinine clearance and concerns about reduced efficacy [30,36,37]. Finally, the correlation observed between the sensitivity of our isolates to antibiotics suggests that antibiotic resistance is a problem that may affect most existing antibacterial, given the overall evolution observed.

\section{Conclusion}

Antibiotic resistance should be continuously evaluated in patients of all ages and in all hospitals where possible, so as to monitor the evolution of this major public health issue. In this investigation performed on UPEC strains isolated from urine samples of children diagnosed with UTIs in Russian Children's Clinical Hospital in Moscow, we found that FO was the only antibiotic to which all UPEC were S. The unusual resistance to IMP and more globally to other antibiotics observed in some strains is alarming and suggests the need for more judicious use of these antibiotics. However, our study has several limitations, especially regarding the lack of information on the subjects' medication profile before their UTIs and the lack of 
investigation of genetic elements related to the resistance.

\section{Authors' Contributions}

SS, BMN, and PIV: Conceptualized and designed the research. SS: Conducted laboratory experiments. MMJA, SS, PIV, VEG, and YNV: Wrote the first manuscript draft, edited and revised the manuscript. All authors critically reviewed and approved the final manuscript.

\section{Acknowledgments}

This publication has been supported by the RUDN University strategic Academic Leadership Program, Russia.

\section{Competing Interests}

The authors declare that they have no competing interests.

\section{Publisher's Note}

Veterinary World (Publisher of International Journal of One Health) remains neutral with regard to jurisdictional claims in published institutional affiliation.

\section{References}

1. Stamm, W.E. and Norrby, S.R. (2001) Urinary tract infections: Disease panorama and challenges. J. Infect. Dis., 183(Suppl 1): S1-S4.

2. Flores-Mireles, A.L., Walker, J.N., Caparon, M. and Hultgren, S.J. (2015) Urinary tract infections: Epidemiology, mechanisms of infection and treatment options. Nat. Rev. Microbiol., 13(5): 269-284.

3. Laupland, K.B., Ross, T., Pitout, J.D.D., Church, D.L. and Gregson, D.B. (2007) Community-onset urinary tract infections: A population-based assessment. Infection, 35(3): 150-153.

4. Raksha, R., Srinivasa, H. and Macaden, R.S. (2003) Occurrence and characterisation of uropathogenic Escherichia coli in urinary tract infections. Indian J. Med. Microbiol., 21(2): 102-107.

5. Foxman, B. (2013) Urinary tract infection syndromes: Occurrence, recurrence, bacteriology, risk factors, and disease burden. Infect. Dis. Clin. North Am., 28(1): 1-13.

6. Asadi, K., Habibi, M.R. and Bouzari, R. (2019) Urinary tract infection: Pathogenicity, antibiotic resistance and development of effective vaccines against uropathogenic Escherichia coli. Mol. Immunol., 108: 56-67.

7. Brumbaugh, A.R., Smith, S.N. and Mobley, H.L. (2013) Immunization with the yersiniabactin receptor, FyuA, protects against pyelonephritis in a murine model of urinary tract infection. Infect. Immunity, 81(9): 3309-3316.

8. Micali, S., Isgro, G., Bianchi, G., Miceli, N., Calapai, G. and Navarra, M. (2014) Cranberry and recurrent cystitis: More than marketing? Crit. Rev. Food Sci. Nutr., 54(8): 1063-1075.

9. Yun, K.W., Kim, H.Y., Park, H.K., Kim, W. and Lim, I.S. (2014) Virulence factors of uropathogenic Escherichia coli of urinary tract infections and asymptomatic bacteriuria in children. J. Microbiol. Immunol. Infect., 47(6): 455-461.

10. Williams, G. and Craig, J.C. (2009) Prevention of recurrent urinary tract infection in children. Curr. Opin. Infect. Dis., 22(1): 72-76.

11. Lob, S.H., Nicolle, L.E., Hoban, D.J., Kazmierczak, K.M., Badal, R.E. and Sahm, D.F. (2016) Susceptibility patterns and ESBL rates of Escherichia coli from urinary tract infections in Canada and the United States, SMART 20102014. Diagn. Microbiol. Infect. Dis., 85(4): 459-465.

12. Morrissey, I., Hackel, M., Badal, R., Bouchillon, S., Hawser, S. and Biedenbach, D. (2013) A review of ten years of the study for monitoring antimicrobial resistance trends (SMART) from 2002 to 2011. Pharmaceuticals, 6(11): 1335-1346.

13. Mahony, M., McMullan, B., Brown, J. and Kennedy, S.E. (2019) Multidrug-resistant organisms in urinary tract infections in children. Pediatr. Nephrol., 35(9): 1563-1573.

14. Bitsori, M., Maraki, S., Kalmanti, M. and Galanakis, E. (2009) Resistance against broad-spectrum $\beta$-lactams among uropathogens in children. Pediatr. Nephrol., 24(12): 2381-2386.

15. Manga, M.J.A, Andreevna, S.L. and Viktorovna, P.I. (2020) Evaluation of apparent microflora and study of antibiotic resistance of coliforms isolated from the shells of poultry eggs in Moscow-Russia. J. Adv. Microbiol., 20(4): 70-77.

16. Clinical and Laboratory Standards Institute. (2021) Control Methods. Biological and Microbiological Factors: Performance Standards for Antimicrobial Susceptibility Testing. $31^{\text {st }}$ ed. Clinical and Laboratory Standards Institute, Wayne, PA.

17. Shirani, K., Seydayi, E. and Boroujeni, K.S. (2019) Prevalence and antibiotic resistance pattern of extended-spectrum beta-lactamase-producing Escherichia coli in clinical specimens. J. Res. Med. Sci., 24(1): 103.

18. Okesola, A.O. and Aroundegbe, T.I. (2011) Antibiotic resistance pattern of uropathogenic Escherichia coli in South West Nigeria. Afr. J. Med. Med. Sci., 40(3): 235-238.

19. Mukherjee, M., Basu, S., Mukherjee, S.K. and Majumder, M. (2013) Multidrug-resistance and extended-spectrum beta-lactamase production in uropathogenic $E$. coli which were isolated from hospitalized patients in Kolkata, India. J. Clin. Diagn. Res., 7(3): 449.

20. Widayati, A., Suryawati, S., de Crespigny, C. and Hiller, J.E. (2011) Self-medication with antibiotics in Yogyakarta City Indonesia: A cross sectional population-based survey. $B M C$ Res. Notes, 4(1): 491.

21. Salama, B., Bavurhe, B.Z. and Kadima, J.N. (2020) Management of acute respiratory infections in children under five by self-medication and prescription of antibiotics in Bukavu. Int. J. Trop. Dis. Health, 40(4): 1-10.

22. Paschke, A.A., Zaoutis, T., Conway, P.H., Xie, D. and Keren, R. (2010) Previous antimicrobial exposure is associated with drug-resistant urinary tract infections in children. Pediatrics, 125(4): 664-672.

23. Arsene, M.M.J, Podoprigora, I.V., Volina, E.G., Ermolaev, A.V. and Smolyakova, L.A. (2021) Evaluation of changes induced in the probiotic Escherichia coli M17 following recurrent exposure to antimicrobials. J. Pharm. Res. Int., 33(29B): 158-167.

24. Uzoechi, S.C. and Abu-Lail, N.I. (2019) The effects of $\beta$-lactam antibiotics on surface modifications of multidrug-resistant Escherichia coli: A multiscale approach. Microsc. Microanal., 25(1): 135-150.

25. Ranjini, C.Y., Kasukurthi, L.R., Madhumati, B. and Rajendran, R. (2015) Prevalence of multidrug resistance and extended-spectrum beta-lactamases among uropathogenic Escherichia coli isolates in a tertiary care hospital in South India: An alarming trend. Community Acquir. Infect., 2(1): 19.

26. Lavigne, J.P., Bruyère, F., Bernard, L., Combescure, C., Ronco, E., Lanotte, P., Coloby, P., Thibault, M., Cariou, G., Desplaces, N., Costa, P. and Sotto, A. (2016) Resistance and virulence potential of uropathogenic Escherichia coli strains isolated from patients hospitalized in urology departments: A French prospective multicentre study. J. Med. Microbiol., 65(6): 530-537.

27. Abd El Ghany, M., Sharaf, H., Al-Agamy, M.H., Shibl, A., Hill-Cawthorne, G.A. and Hong, P.Y. Genomic characterization of NDM-1 and 5, and OXA-181 carbapenemases in 
uropathogenic Escherichia coli isolates from Riyadh, Saudi Arabia. PLoS One, 13(8): e0201613.

28. Mbarga, M.J.A., Podoprigora, I.V., Anyutoulou, K.L.D., Esther, N. and Senyagin, A.N. (2021) Urinary tract infections (UTIs): Virulence factors, resistance to antibiotics and management of uropathogenic (UP) bacteria with medicinal plants a review. J. Appl. Pharm. Sci., 11(7): 1-12.

29. Czajkowski, K., Broś-Konopielko, M. and TeligaCzajkowska, J. (2021) Urinary tract infection in women. Prz. Menopauzalny, 20(1): 40-47.

30. DeMarsh, M., Bookstaver, P.B., Gordon, C., Lim, J., Griffith, N., Bookstaver, N.K. and Al-Hasan, M.N. (2020) Prediction of trimethoprim/sulfamethoxazole resistance in community-onset urinary tract infections. J. Glob. Antimicrob. Resist., 21: 218-222.

31. Eliopoulos, G.M. and Huovinen, P. (2001) Resistance to trimethoprim-sulfamethoxazole. Clin. Infect. Dis., 32(11): 1608-1614.

32. Blahna, M.T., Zalewski, C.A., Reuer, J., Kahlmeter, G., Foxman, B. and Marrs, C.F. (2006) The role of horizontal gene transfer in the spread of trimethoprim-sulfamethoxazole resistance among uropathogenic Escherichia coli in Europe and Canada. J. Antimicrob. Chemother., 57(4): 666-672.

33. Hirsch, E.B., Raux, B.R., Zucchi, P.C., Kim, Y., McCoy, C., Kirby, J.E., Wright, S.B. and Eliopoulos, G.M. (2015)
Activity of fosfomycin and comparison of several susceptibility testing methods against contemporary urine isolates. Int. J. Antimicrob. Agents, 46(6): 642-647.

34. Kresken, M., Körber-Irrgang, B., Biedenbach, D.J., Batista, N., Besard, V., Cantón, R., García-Castillo, M., Kalka-Moll, W., Pascual, A., Schwarz, R., Van Meensel, B., Wisplinghoff, H. and Seifert, H. (2016) Comparative in vitro activity of oral antimicrobial agents against Enterobacteriaceae from patients with community-acquired urinary tract infections in three European countries. Clin. Microbiol. Infect., 22(1): 63.e1-63.e5.

35. Dijkmans, A.C., Zacarías, N.V.O., Burggraaf, J., Mouton, J.W., Wilms, E., van Nieuwkoop, C., Touw, D.J., Stevens, J. and Kamerling, I.M.C. (2017) Fosfomycin: Pharmacological, clinical and future perspectives. Antibiotics (Basel), 6(4): 24.

36. Matthews, P.C., Barrett, L.K., Warren, S., Stoesser, N., Snelling, M., Scarborough, M. and Jones, N. (2016) Oral fosfomycin for treatment of urinary tract infection: A retrospective cohort study. BMC Infect. Dis., 16(1): 1-11.

37. Huttner, A., Kowalczyk, A., Turjeman, A., Babich, T., Brossier, C., Eliakim-Raz, N. and Harbarth, S. (2018) Effect of 5-day nitrofurantoin vs single-dose fosfomycin on clinical resolution of uncomplicated lower urinary tract infection in women: A randomized clinical trial. JAMA, 319(17): 1781-1789.

\section{$* * * * * * * *$}

